\title{
Beyond Information Overload and Stress: A Plea to Acknowledge the Complexity of Problematic Internet Use and Consider Individualized Intervention Strategies
}

\author{
Maèva Flayelle ${ }^{1}$, Vladan Starcevic ${ }^{2}$ and Joël Billieux ${ }^{1,3, *}$ \\ ${ }^{I}$ Addictive and Compulsive Behaviours Lab (ACB-Lab), Institute for Health and Behaviour, University of Luxembourg, \\ Esch-sur-Alzette, Luxembourg, ${ }^{2}$ Discipline of Psychiatry, Nepean Clinical School, Sydney Medical School, Faculty of \\ Medicine and Health, University of Sydney, Sydney, NSW, Australia; ${ }^{3}$ Addiction Division, Department of Mental Health \\ and Psychiatry, University Hospitals of Geneva, Geneva, Switzerland
}

Commentary on: Addictive influences and stress propensity in heavy internet users: A proposition for information overload mediated neuropsychiatric dysfunction (Kumar et al., 2017)

At a time when internet overuse is increasingly investigated, with its addictive attributes being at the heart of scientific debates [1-3], Kumar et al. [4] suggest that this behaviour is a serious public health problem that requires "deaddiction" measures. Relying on a symptom-based approach that emphasizes "stress" apparently caused by internet overuse, Kumar et al. [4] make a number of aetiological, diagnostic and preventive/curative claims which are, in our view, overly general and simplistic. The present commentary critically examines these claims.

Kumar et al. [4] assert that internet overuse is a "syndrome similar to the addiction of drug and substances of abuse". They state that the clinical manifestations of internet overuse "may be milder" than those of substance addictions and that they include withdrawal. This reflects a reductionist biomedical approach to problematic use of technologies, which has been strongly criticized by scholars, including those who specialize in addiction research [5-8]. Such an approach is inadequate because of its focus on the surface, symptom manifestations, unjustified imposition of the substance addiction framework on problematic internet use, conceptual shortcuts that may result in inappropriate treatment strategies and the risk of pathologizing normal behaviours $[5,7,9]$.

In addition, Kumar et al. [4] repeatedly refer to "heavy internet use" and "compulsive internet overuse" without defining these terms and without making an effort to distinguish between them. Although they mention internet overuse for "general purposes" and "specialized categories of internet overuse problems", it is not clear whether and how they differ and the overall impression is that heavy internet use/compulsive internet overuse is considered a discrete clinical entity. This neglects the type of excessive/problematic online behaviour

*Address correspondence to this author at the Addictive and Compulsive Behaviours Lab, Institute for Health and Behaviour, Campus Belval, Maison des Sciences Humaines, 11, Porte des Sciences, L-4366 Esch-sur-Alzette, Luxembourg; Tel: +35246664439207; E-mail: joel.billieux@uni.lu exhibited (e.g., video gaming, gambling, shopping, cybersex, use of social networking sites or streaming services) and thereby espouses a « unitary position » to problematic internet use [10]. This position is now considered misleading and outdated because of the heterogeneity of the concept of problematic internet use. Indeed, this concept is an "umbrella entity" [10-16] encompassing a variety of behaviours, with the internet as a "delivery mechanism" perhaps the only feature they have in common [10]. The Spectrum Hypothesis of problematic online behaviours posits that they should be conceptualised within a spectrum of related, yet distinct disorders, which are associated with both common and unique etiological factors $[17,18]$. This hypothesis recently received empirical support [19], further cementing the need to focus on the specific problematic behaviours mediated by the internet $[17,18,20]$.

As the medium (i.e., the internet) is the focal point of the reasoning by Kumar et al. [4], there is a heavy emphasis on "information overload" or "information fatigue" caused by internet overuse. According to Kumar et al. [4], this phenomenon is the presumed mechanism that accounts for the negative consequences of excessive internet use, conceptualised in terms of "mental strain" and "stress". Such an approach is rooted in a reception model that implies that certain characteristics of media/technologies have a unidirectional and universal impact on users/recipients [21]. However, this model is considered outdated as it is simplistic and fails to grasp the breadth of media effects. Indeed, media effects are first and foremost contingent upon individual differences [21] and result from complex, intertwined processes (e.g., cognitive, affective, motivational, developmental, social) $[21,22]$, as stipulated by prominent media effects theories [23-26]. In accordance with a more nuanced perspective on media effects, the focus should move to various underlying mechanisms and mediators and moderators of the effects of both the internet and internet-mediated excessive behaviours. This should help better understand internet use and problematic internet-related behaviours. 
Whilst emphasizing "information overload" or "information fatigue", Kumar et al. [4] seem to blame the medium, i.e., the internet and the associated factors (e.g., "screen facing time, display characteristics of the device and multitasking attempts of the user") for the negative effects of excessive internet use. These effects include "psychological disturbances" such as anxiety, impulsivity and irritation. Kumar et al. [4] barely take into account a well-established fact that various psychopathological factors, e.g., social anxiety, depression and attention-deficit/hyperactivity disorder, may precede and lead to excessive or problematic internet use, whereby its negative effects can hardly be attributed solely to the internet. In addition, evidence is lacking that "information overload" or "information fatigue" is a crucial mechanism involved in problematic online gaming, gambling, shopping, sexual activities, social networking or use of streaming services - activities usually implicated in problematic internet use.

The model of "stress" that Kumar et al. [4] propose as an explanation for the effects of excessive internet use is too broad and unhelpful, given the frequently elusive, nonspecific connotations of the concept of stress. Likewise, a recommendation by Kumar et al. [4] to use stress reduction techniques as the key treatment strategy for problematic internet use is also too broad and "generic". Moreover, this recommendation appears to be of a limited clinical relevance because of the questionable effectiveness of treatment interventions that target symptoms (i.e., "stress" and its manifestations) rather than causes and the underlying processes [9, 27]. The best alternative to the non-specifically applied symptom-based approach is a person-centred strategy that identifies and addresses the unique (psychological) processes that underlie specific excessive online behaviours in each individual $[5,7,28]$. Such an in-depth understanding is a prerequisite for designing adequate prevention and intervention strategies for problematic internet-mediated behaviours.

\section{REFERENCES}

[1] Kardefelt-Winther D. A conceptual and methodological critique of internet addiction research: Towards a model of compensatory internet use. Comput Human Behav 2014; 31: 351-4.

[2] Bergmark KH, Stensson E, Bergmark A. Internet addiction: The making of a new addiction. Jacobs J Addict Ther 2016; 3: 1-22.

[3] Kuss DJ, Billieux J. Technological addictions: Conceptualisation, measurement, etiology and treatment. Addict Behav 2017; 64: 2313.

[4] Kumar A, Faiq MA, Pandey SN, et al. Addictive influences and stress propensity in heavy internet users: A proposition for information overload mediated neuropsychiatric dysfunction. Curr Psychiatry Rev 2017; 13: 293-300.

[5] Billieux J, Schimmenti A, Khazaal Y, Maurage P, Heeren A. Are we overpathologizing everyday life? A tenable blueprint for behavioral addiction research. J Behav Addict 2015; 4: 119-23.

[6] James RJE, Tunney RJ. The need for a behavioural analysis of behavioural addictions. Clin Psychol Rev 2016; 52: 69-76.
[7] Kardefelt-Winther D, Heeren A, Schimmenti A, et al. How can we conceptualize behavioural addiction without pathologizing common behaviours? Addiction 2017; 112: 1709-15.

[8] Starcevic V. Behavioural addictions: A challenge for psychopathology and psychiatric nosology, Aust N Z J Psychiatry 2016; 50: 721-5.

[9] Billieux J, Philippot P, Schmid C, Maurage P, de Mol J, van der Linden M. Is dysfunctional use of the mobile phone a behavioural addiction? Confronting symptom-based versus process-based approaches. Clin Psychol Psychother 2015; 22: 460-8.

[10] Starcevic V, Aboujaoude E. Internet addiction: Reappraisal of an increasingly inadequate concept. CNS Spectr 2017; 22: 7-13.

[11] Andreassen CS, Billieux J, Griffiths MD, et al. The relationship between addictive use of social media and video games and symptoms of psychiatric disorders: A large-scale cross-sectional study. Psychol Addict Behav 2016; 30: 252-262.

[12] Király O, Griffiths MD, Urbán R, et al. Problematic internet use and problematic online gaming are not the same: Findings from a large nationally representative adolescent sample. Cyberpsychol Behav Soc Netw 2014; 17: 749-54.

[13] Yellowlees PM, Marks S. Problematic internet use or internet addiction? Comput Human Behav 2007; 23: 1447-53.

[14] Northrup J, Lapierre C, Kirk J, Rae C. The internet process Addiction Test: Screening for addictions to processes facilitated by the internet. Behav Sci 2015; 5: 341-52.

[15] Laconi S, Tricard N, Chabrol H. Differences between specific and generalized problematic internet uses according to gender, age, time spent online and psychopathological symptoms. Comput Human Behav 2015; 48: 236-44.

[16] Pawlikowski M, Nader IW, Burger C, Biermann I, Stieger S, Brand M. Pathological internet use - it is a multidimensional and not a unidimensional construct. Addict Res Theory 2014; 22: 166-75.

[17] Starcevic V, Billieux J. Does the construct of internet addiction reflect a single entity or a spectrum of disorders? Clin Neuropsy 2017; 14: 5-10.

[18] Billieux J. Problematic mobile phone use: A literature review and a pathways model. Curr Psychiatry Rev 2012; 8: 299-307.

[19] Baggio S, Starcevic V, Studer J, et al. Technology-mediated behaviors constitute a spectrum of related yet distinct conditions: A network perspective. Psychol Addict Behav 2018; 32: 564-72.

[20] Morahan-Martin J. Internet Abuse: Addiction? Disorder? Symptom? Alternative Explanations? Soc Sci Comput Rev 2015; 23: 39-48.

[21] Valkenburg PM, Peter J, Walther JB. Media effects: Theory and research. Annu Rev Psychol 2016; 67: 315-38.

[22] Dill KE. The Oxford handbook of media psychology. New York: Oxford University Press 2013.

[23] Rubin A. Uses-and-gratifications perspective on media effects. In: Bryant J, Oliver MB, Eds. Media effects: Advances in theory and research. New York: Routledge 2009; pp. 165-84.

[24] Klapper JT. The effects of mass communications. Oxford, England: Free Press of Glencoe 1960.

[25] Slater MD. Reinforcing spirals: The mutual influence of media selectivity and media effects and their impact on individual behavior and social identity. Commun Theor 2007; 17: 281-303.

[26] Valkenburg PM, Peter J. The differential susceptibility to media effects model. J Commun 2013; 63: 221-43.

[27] Wéry A, Schimmenti A, Karila L, Billieux J. Where the mind cannot dare: A case of addictive use of online pornography and its relationship with childhood trauma. J Sex Marital Ther 2018; 22: 114.

[28] Flayelle M, Maurage P, Vögele C, Karila L, Billieux J. Time for a plot twist: Beyond confirmatory approaches to binge-watching research. Psychol Pop Media Cult 2018 [Epub ahead of print]. 